\title{
Trust-wide improvement of discharge summaries at University Hospital Southampton
}

\author{
Author: Clare Smith*
}

\section{Background}

The discharge summary is a document completed by hospital staff at the end of an admission to communicate inpatient information and ongoing plans with community care providers and the patient. The electronic discharge summary at University Hospital Southampton was developed 10 years ago. Following annual review of both national standards and local requirements it has grown considerably. This has substantially increased the content and therefore it takes a long time to complete, and is challenging for general practitioners (GPs) and community services to decipher relevant information. The information for patients is not clear. It is neither patient-centred nor clinician-friendly, and is therefore not fit for purpose. A review is required to ensure the discharge summary is suitable for patients, providers and users.

\section{Method}

I am undertaking this project with a multidisciplinary team utilising the IHI 'Model for Improvement', which asks three questions: What are we trying to accomplish? How will we know that a change is an improvement? What change can we make that will result in an improvement? followed by 'Plan, Do, Study, Act' (PDSA) cycles.

We have engaged multiple stakeholders in order to fully understand the problem from different perspectives. This includes patients, commissioners, and both clinicians and non-clinicians across primary and secondary care. Process mapping has helped identify where bottlenecks occur and a driver diagram influenced the scope and spectrum of PDSA cycles.

\section{The current situation}

University Hospital Southampton produces 100,000 discharge summaries a year, $80 \%$ of which are completed by junior doctors. There is variation in the number completed depending on hospital specialty. The discharge summary template is the same regardless of the department or clinical need. A blank discharge summary with just headings will print onto four sheets of paper. All headings within the document will be printed in the final summary regardless of whether they have been populated or are relevant.
The discharge summary takes junior doctors 20-40 minutes to complete and within some specialties, it takes $60-70 \%$ of their day. On one-in-four occasions the IT system crashes and does not save any data entered. There is a negative culture among junior doctors towards the discharge summary which has had an impact on their completeness and accuracy. This is evidenced from coders who use the discharge summary to code admission episodes. We have had negative feedback from GPs who find discharge summaries incredibly frustrating, and some report they are employing people to decipher the relevant information. Patients have raised formal complaints and there are many calls to the Medicine Information Service with questions or queries.

\section{Current PDSA cycles}

> Patient-facing and friendly

$>$ A multidisciplinary document that moves away from a letter from hospital doctor to GP

> Negotiations with commissioners for the content of the summary

> Appreciative inquiry methodology to create a culture change and an ideal model

$>$ Working with IT to develop the new prototype

> Use of voice recognition and auto-population of fields. 